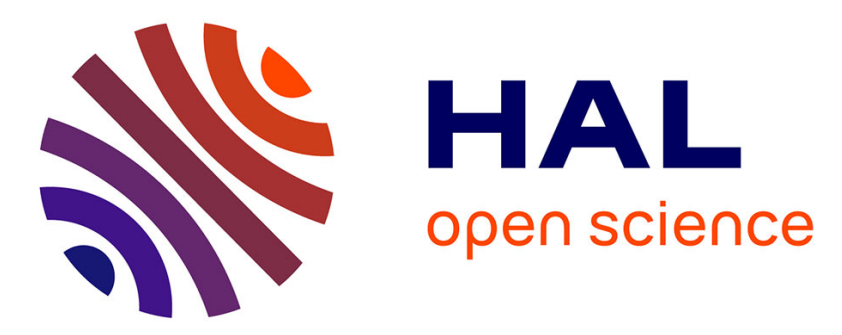

\title{
High-power laser delocalization in plasmas leading to long-range beam merging
}

M. Nakatsutsumi, J.-R Marquès, P. Antici, N. Bourgeois, J. L Feugeas, T. Lin, Ph Nicolaï, L. Romagnani, R. Kodama, P. Audebert, et al.

\section{To cite this version:}

M. Nakatsutsumi, J.-R Marquès, P. Antici, N. Bourgeois, J. L Feugeas, et al.. High-power laser delocalization in plasmas leading to long-range beam merging. Nature Physics, 2010, 6 (12), pp.10101016. 10.1038/nphys1788 . hal-03321060

\section{HAL Id: hal-03321060 \\ https://hal.science/hal-03321060}

Submitted on 17 Aug 2021

HAL is a multi-disciplinary open access archive for the deposit and dissemination of scientific research documents, whether they are published or not. The documents may come from teaching and research institutions in France or abroad, or from public or private research centers.
L'archive ouverte pluridisciplinaire HAL, est destinée au dépôt et à la diffusion de documents scientifiques de niveau recherche, publiés ou non, émanant des établissements d'enseignement et de recherche français ou étrangers, des laboratoires publics ou privés. 


\title{
High-power laser delocalization in plasmas leading to long-range beam merging
}

\author{
M. Nakatsutsumi ${ }^{1,2}$, J.-R. Marquès ${ }^{1}$, P. Antici ${ }^{1}$, N. Bourgeois ${ }^{1}$, J. L. Feugeas ${ }^{3}$, T. Lin ${ }^{4}$, Ph. Nicolaï ${ }^{3}$, \\ L. Romagnani ${ }^{1}$, R. Kodama ${ }^{2}$, P. Audebert ${ }^{1}$, and J. Fuchs ${ }^{1 *}$ \\ ${ }^{1}$ LULI, Ecole Polytechnique, CNRS, CEA, UPMC, Route de Saclay, 91128 Palaiseau, France \\ ${ }^{2}$ Graduate School of Engineering, Osaka University, 2-1 Yamada-oka, Suita, Osaka 565-0871, Japan \\ ${ }^{3}$ Centre Lasers Intenses et Applications, Université Bordeaux 1-CNRS-CEA, 33405 Talence Cedex, \\ France \\ ${ }^{4}$ Fox Chase Cancer Center, Philadephia, USA
}

*julien.fuchs@polytechnique.fr

PACS numbers: 42.65.Sf, 52.35.Mw, 52.38.Hb, 47.35.-i

Attraction and fusion between co-propagating light beams, mutually coherent or not, can take place in nonlinear media due to the beam power modifying the refractive index of the medium. In the context of high-power light beams, induced modifications of the beam patterns could potentially impact many topics ranging from long range laser propagation, the study of astrophysical colliding blast waves, or to inertial confinement fusion (ICF). Here, through experiments and simulations, we show that in a fully ionized plasma, which is a nonlinear medium, beam merging can take place for high-power and mutuallyincoherent beams that are initially separated by several beam diameters. This is in contrast with the usual assumption that this type of interaction is limited to beams separated by only one beam diameter. This effect, which is orders of magnitude more significant than Kerr-like nonlinearity in gases, demonstrates the importance of potential cross-talk amongst multiple beams in plasma.

For several decades, beam coupling phenomenon have been observed in non-ionized media $[1,2,3,4]$. The nonlinearity responsible for these can be local, in which case the interaction is limited to neighbor beams, defined here as beams for which their separation equals their initial diameter, or nonlocal in which case the separation is larger [5,6]. Local nonlinearity in plasma due to refractive index change induced by the beam power is well-known and can lead to interaction between neighbor beams [7,8]. Here the unexpected nonlocal nonlinearity that is observed in fully ionized plasmas exhibits a different physical origin. Here, two different effects play a key role: (i) laser beam delocalization (i.e. beam transverse motion or "hosing" and spreading) [9] and (ii) hydrodynamic boring of the plasma. These effects require 
long (100's of ps long) laser pulses to be effective since they take place on acoustic time-scales. However, they allow strong nonlocal interaction between far-distant high-power laser beams. It is precisely because of the previously neglected beam delocalization that interactions were up to now assumed to be limited to neighbor beams $[10,11,12,13,14]$.

Experimentally, the issue had remained also poorly understood, as (i) usually high-power long pulse lasers have poor wavefront quality and hence do not allow using few, well-controlled light filaments, and (ii) resorting to smoothed beams (i.e. composed of many beamlets) [15], where many mutual interactions take place, makes it difficult to isolate individual processes. In the present study, we uniquely access the dynamics of single and dual beams thanks to (i) the use of well-controlled, close to diffraction limited "single hot spot" beams $[16,17,18,19]$, and (ii) a novel diagnostic allowing uniquely time-resolved and two-dimensional (2D) space-resolved transverse imaging of the transmitted beam. These are key techniques that allowed us to understand the dynamics of a single beam and of multiple beam coupling in plasmas.

We first address the dynamics of a single high-power ( $20 \mathrm{GW}, 60 \mu \mathrm{m}$ FWHM, $400 \mathrm{ps}, 3 \times 10^{14}$ $\left.\mathrm{Wcm}^{-2}, \lambda=1.05 \mu \mathrm{m}\right)$ ) beam propagating in a gas jet (see Methods) as it governs the coupling dynamics of two parallel beams. Such high-power ensures that the plasma nonlinearity is strongly excited, as is the case for realistic ICF beams [37]. As observed by the interferometry diagnostic (see Methods), the He plasma in which the beam propagates is fully ionized. This is due to multiphoton ionization taking place around -300 ps (i.e. 300 ps before the peak of the pulse), followed by avalanche collisional ionization [20]. The CHIC hydrodynamic simulations performed with the present experimental parameters (see below) yield temperatures in the $200-400 \mathrm{eV}$ range, which is consistent with measurements of previous experiments performed under similar conditions [21,22].

After ionization takes place, our diagnostic, which monitors temporal snapshots (integrated over $30 \mathrm{ps}$ ) of the transverse beam profile in the plasma, witnesses strong delocalization of the laser beam (see Fig. 1(b)) induced by the plasma nonlinearity. There are two manifestations of the delocalization: (i) motion of the beam centroid in its transverse plane and (ii) beam spreading. Note that, comparatively, the beam with the same power propagating in vacuum does not display such dynamics and was observed, as expected, to stay at the same location, with the same size, in the image plane (see Fig.1(a)).

Single beam motion in plasma had only been theoretically predicted $[23,24,25,26]$ but not previously observed in experiments. This is due to the 1D geometry of the time-resolved diagnostics used thus far which could only analyze a fixed lineout in the image plane and could not resolve beam mo- 
tion. Numerical simulations have revealed that beam motion results from a resonant instability between the localized beam and the excited eigenmodes of the depleted plasma density channel in which the high-power beam propagates. Such a channel is observed in the experiment (see Fig.3) by the interferometry diagnostic. In our high-power, long-pulse conditions, the plasma channel is generated by the laser beam through thermal pressure due to the laser light deposited heat [27], as supported by the numerical simulations of the laser-plasma coupling performed using the experimental parameters (see below). Note that the observed large radial amplitude of the motion ( 70 microns), being on the order of the initial beam diameter itself, is well consistent with the theoretical predictions of beam motion.

On top of moving transversally, the beam propagating in the plasma is also seen, in an initial temporal phase, to spread in its transverse plane (see Fig. 1(c)). The beam spreading increases with the background plasma density, as illustrated in Fig. 1(d). Such spreading, already witnessed in experiments using smoothed beams composed of many individual beamlets [28], is observed in simulations to originate from a combination of stimulated Brillouin forward scattering (SBFS) and filamentation [24,28,29,30,31] triggered at high-power. Indeed, ionization induced refraction [32] is not likely to occur in our experimental conditions as the same spreading was observed when pre-ionizing the He gas by an auxiliary beam. Beam motion and beam spreading are intrinsically coupled [24] as radiation associated with filamentation seeds the beam motion instability; and as beam motion and radiation leakage through the walls of the hosing density channel excite plasma ion waves and seed SBFS and filamentation. After this initial phase, around the temporal peak of the pulse, we see a reduction of the beam spatial spread (see Fig. 1(c)) due to beam self-trapping [33] in the density channel focusing structure (an on-axis density minimum corresponding to an on-axis peak of refractive index $N$ as $\left.N=\left(1-n_{e} / n_{c}\right)^{1 / 2}\right)$. This phase is delayed compared to the defocusing phase as the plasma ionization and the channel formation happen on different time-scales [21], the latter taking place at the slower sound speed $\mathrm{c}_{\mathrm{s}} \sim 0.1 \mu \mathrm{m} / \mathrm{ps}$.

Now, when co-propagating two beams which are initially either close to each other or far apart (i.e. separated by several vacuum spot diameters), we observe clear and striking features in the temporal behavior of the overall light pattern; i.e. we observe beam merging even for far-distant beams, provided that the background plasma density is high enough. This is different from the vacuum-propagation case where, as expected, two separated beams do not interact (see Fig.1(a)). Beam merging in the plasma results from two physical mechanisms: first, the merging of the two density channels bored by the two beams, second, when this hydrodynamic merging is achieved, the merging of the two beams within it. This is made possible by the fact that a single merged channel acts as a common overall focusing struc- 
ture. Similarly as a lens, it allows the beams to be brought closer to each other. Numerical simulations (see Methods) performed in the conditions of the experiment reveal that the beam delocalization (motion and spreading) observed for a single beam, although not accounted so far when considering coupled beams, plays a key role in allowing merging of the individual plasma channels and subsequent beam merging.

Figure 2(a) shows that, as assumed up to now, two far-distant beams, i.e. having an initial mutual distance of $\mathrm{d}=300 \mu \mathrm{m}(=5 \times$ vacuum spot size $)$, behave independently when propagated in plasma having a density of $\mathrm{n}_{\mathrm{e}}=0.016 \mathrm{n}_{\mathrm{c}}\left(\mathrm{n}_{\mathrm{c}}=10^{21} \mathrm{~cm}^{-3}\right)$. Consistently, as shown in Fig.3(a), the transverse interferometry for the same shot reveals the existence of an on-axis density peak (see the central kink in the fringes in Fig.3(a)) in between the two individual channels dug by the two beams. This prevents the beams from interacting. Note that the diameter of each of the two channels $(\sim 300 \mu \mathrm{m}$ between the channel-edge density peaks) is much larger than the initial beam size $(60 \mu \mathrm{m})$ due to combined effect of beam delocalization (that extends the area of which laser energy is deposited in the plasma) and of hydrodynamic expansion of the channel edges (taking place at $\mathrm{c}_{\mathrm{s}}$ ).

However, as shown in Fig.2(b), when the distance was reduced to $d=150 \mu \mathrm{m}(=2.5 \times$ vacuum spot size) or lower values, the initially distinct beams are observed to coalesce into a single beam around the temporal peak. Simultaneously, as witnessed by Fig.3(b) [which corresponds to the same shot as shown in Fig.2(b)], we also observe a merging of the individual plasma density channels into a single broad density channel. The merging process is stable from shot-to-shot despite realignement and small (few ps) delay changes, hinting that it does not depend on particular phase conditions, i.e. that it is an incoherent process.

Numerical simulations, performed with CHIC in 2D in the plane transverse to the propagation, shed light on the mechanism leading to the merging of the plasma channels into a single structure. In the simulations, two parallel beams deposit their energy at locations corresponding to the beam separation (either 150 or $300 \mu \mathrm{m}$, as in the experiment). The beam propagation (along the third spatial dimension) cannot be directly accounted for due to computing constraints. We nonetheless take the effect of beam propagation into account by parametrizing each beam's so that (i) the correct temporal evolution of the laser energy and (ii) the correct spatial distribution of the deposited energy are accounted for. Beam delocalization is taken into account through a modification of the beam radius as well as of its centroid location. The beam motion (see Fig.1(b)) is modeled as an oscillation with an amplitude of $\sim 70 \mu \mathrm{m}$ and a period of $\sim 200$ ps while the beam spreading is modeled according to 
Fig.1(c). This way, although the simulation is made only in a 2D transverse plane, we can integrate the effect of beam propagation in the simulation.

As shown in Fig.4(a), we observe in the simulations that for an initial beam separation of $300 \mu \mathrm{m}$, two plasma density channel are formed. This is consistent with the experimental observation of Fig.3(a). When reducing the beam separation down to $150 \mu \mathrm{m}$, we observe in the simulations that the initially separated channels merge around the temporal peak of the laser pulses (see Fig.4(b)), again consistently with the experimental observation of Fig.3(b). In this latter case, the mechanism of the removal of the plasma density local maximum in between the two beams is clarified in Fig.4(c-d) where we observe that the plasma is expelled by each beam from its axis. As it collides with the plasma produced by the other beam, it is deflected sideways (Fig.4(c)). Then a central density depression is formed (Fig.4(d)). The effect of thermal pressure is dominant over the light pressure (i.e. ponderomotive force) for the hydrodynamic evolution of the plasma: as shown in Fig.4(b). Simulations performed with and without the effect of the light pressure show that this effect is negligible. Note that, in order to reproduce the experimental results, the full beam delocalization (i.e. coupled beam spreading and motion) is essential to be taken into account, as simulations performed without either spreading or motion, or without both cannot account for the formation of the central plasma channel observed in the experiment. Both effects reinforce the reduction of the effective beam separation until it is close to the effective beam size (see Fig. 2 (b2)).

Once the single broad channel is formed, it acts like an overall focusing structure and thus favors the merging of the two beams (which can add in intensity, having become incoherent [28]). As shown in Fig.3(d), modeling was done by performing simple ray-tracing calculations of the propagation of light rays within the central density depletion of Fig.3(c) and using as input a plane wave having the size of the two beams side-by-side, $150 \mu \mathrm{m}$ separated, before merging. We find that over the effective $\sim 1500 \mu \mathrm{m}$ propagation distance, the overall beam FWHM is indeed reduced to a size comparable with the observed merged beam size (shown in Fig.2(b4)). Note that this phenomenon of merging through a modification of the medium refractive index having an on-axis maximum is identical to the one predicted for shorter pulses (where the interaction occurs through non-linear index changes instead of hydrodynamics as is the case here) $[8,14]$. We finally stress that several mechanisms add up to allow long-range beam merging: (1) without beam delocalization due to the plasma nonlinearity, the individual beams could not deposit their energy far from their initial location, (2) without the hydrodynamic motion of the plasma, the individual channels could not merge to induce relocalization of light beams into a single, central, light guiding structure, and (3) without the for- 
mation of this focusing plasma density depression, the two beams would just overlap and not reduce to the observed small single hot spot (as shown in Fig.2(b4)).

When we increased the plasma density, we clearly observed the merging of the two beams that behaved independently at lower density. This is illustrated in Fig.5(a-d) which shows the dynamics of two beams with $n_{e}=0.032 n_{c}$ (twice the density of Fig.2(a) for a similar separation $\mathrm{d}=350 \mu \mathrm{m}$ ). This is due to the increased individual beams delocalization (compared to Fig.2(a)) with density (see Fig.1(d)). This will generate heat deposition over a larger zone in the plasma compared to when the density was lower, and it will reduce the effective separation to the effective beam size (see Fig.5(c)). As a result, the two individual channels can mix even though the mutual distance is larger than the lower density case. Figure 5(e) summarizes the maximum mutual beam distance for which we still observe long-range beam merging: clearly it reduces when the plasma density decreases.

When we reduced the plasma nonlinearity by reducing the beam power down to $10 \mathrm{MW}$ (which corresponds to an intensity of the beams of $10^{11} \mathrm{~W} . \mathrm{cm}^{-2}$ ), we observed that beam merging did not take place anymore except for neighbor beams. This can be understood as reducing the laser power (i) inhibits beam delocalization (as we observed: there was no spreading nor transverse motion in this case as the plasma nonlinearity is not triggered), and (ii) reduces $T_{e}$ and thus both the channel expansion speed and the channel depth, thereby preventing merging at long distances. Nonetheless, local plasma heating can still take place and individual channels can form. The CHIC simulations show that in this case the channels can merge in a timescale comparable to the beam duration, but only for neighbor beams. In this case, the mechanism leading to channel formation, expansion and eventually merging is still the thermal pressure, as in the higher laser power, higher plasma temperature case.

To quantify the beam size reduction resulting from beam convergence in the single broad channel at high intensity, we computed the 2D spatial autocorrelation function of the time-resolved 2D images (see Methods). We apply this calculation to three different situations: (i) experimentally observed images when two beams were propagated in plasma, (ii) synthetic images obtained by mathematically adding the image of a single beam (propagating in the same conditions, in the same plasma) to itself spatially shifted, and (iii) synthetic images obtained the same way as for (ii) but this time for linearly-diffracting beams, i.e. beams propagating in vacuum (without plasma). The calculated beam(s) size in the three cases is shown in Fig. 6 for various separations (d) and plasma densities. We clearly observe that beam coupling that leads to beam size reduction takes place even for non-neighbor beams (i.e. for $\mathrm{d} \sim 150 \mu \mathrm{m}$ if $\mathrm{n}_{\mathrm{e}}=0.016 n_{c}$, see Fig. 6(a), and for $\mathrm{d} \sim 350 \mu \mathrm{m}$ if $\mathrm{n}_{\mathrm{e}}=0.032 n_{c}$, see Fig. $6(\mathrm{~b})$ ). The signature of this effect is 
that the coupled beam size is strongly reduced compared to the size of two superimposed, uncoupled (but still propagating in the plasma) beams. We can see here that the point of Fig. 6(a) at $150 \mu$ m separation corresponds quite well to the ray-trace calculation of Fig.3(d): the FWHM is reduced from $2 \times 75 \mu \mathrm{m}$ down to $2 \times 60 \mu \mathrm{m}$ after propagating through the plasma. We also see that the merged beam size $(>60 \mu \mathrm{m}$ in all conditions) is close or larger than the size of two linearly-diffracting beams, which is $60 \mu \mathrm{m}$ FWHM over the interaction length.

Beam energy losses are associated with the combined processes that drive beam merging. Losses suffered by the high-power beam result from ionization, absorption (i.e. plasma heating), and nonlinear filamentation and scattering (either forward or backward). In agreement with analytical estimates, the numerical simulations of CHIC show that ionization and absorption, which induce the plasma channel formation, reduce the beam energy by $\leq 1 \%$. We observe however that beam transmission $\mathrm{T}$ is strongly reduced when the plasma density increases $\left(\mathrm{T}=75 \%\right.$ for $\mathrm{n}_{\mathrm{e}}=0.016 n_{c}$ and $\mathrm{T}=15 \%$ for $\left.\mathrm{n}_{\mathrm{e}}=0.032 n_{c}\right)$, consistently with simple analytical estimates of nonlinear plasma backscattering instabilities developing in the plasma [34]. The latter thus impose strong losses on the propagating beam that are not directly "useful" for the channel formation, but since they induce beam delocalization, they are nonetheless essential to the long-distance merging ability of the plasma medium studied here.

The mechanism deciphered here leading to long-range beam merging could potentially be applied to merge more than two beams. If there is a perfect symmetry between the beams (e.g. three beams at the tips of an equilateral triangle) we can expect that the merging of all beams will take place at the same time. If the beams are positioned asymmetrically, closer beams will merge first while merging of further distant beams will be delayed. In all cases, we can expect the formation of a central density channel that provides a unique guiding structure for the initially separated beams. One can note that high-power long-duration laser beams used for inertial confinement fusion (ICF) $[35,36]$ present a structure where many sub-beamlets compose the overall laser beam. Although the plasma and lasers scales explored here differ from the ones used in ICF, our study suggests that mutual interactions between the beamlets could generate strong localized filaments, exacerbating the detrimental coupling of the laser with long underdense plasmas surrounding the ICF target [35,37], and ultimately damaging fuel compression. To evaluate the importance of these effects, efforts in multi-scale modeling able to resolve the spatial and temporal scales of both the large beam envelope and the individual filament within, as has been shown recently to be achievable with the assistance of massive computing [37], would be required. 


\section{Acknowledgements}

We acknowledge the expert support of the LULI teams and fruitful discussions with F. Amiranoff, L. Bergé, S.N. Chen, S. Hüller, M. Grech, S. Weber, J.P. Zou and Y. Sakawa. This work was supported by grant E1127 from Région Ile-de-France and by the CEA-EURATOM association as an IFE "keep-intouch" activity. M.N. was partially supported by JSPS Postdoctoral Fellowships for Research Abroad.

M.N. (on leave from Osaka University, supervised by R.K.), J.R.M., P.A., N.B., T.L., L.R., J.F. performed the experiments, M.N., J.R.M., J.F. analyzed the data and wrote the paper with discussions with R.K. and P.A., J.L.F and P.N. performed the numerical simulations, J.F. planned the project. 


\section{Methods}

The experiment was performed using the 100 TW laser facility at the Laboratoire pour l'Utilisation des Lasers Intenses (LULI). A laser pulse of $\sim 20 \mathrm{~J}\left(\lambda=1.05 \mu \mathrm{m}, \tau_{\mathrm{L}}=400 \mathrm{ps}\right.$ FWHM duration, horizontally polarized) was focused at a maximum intensity of $\sim 3 \times 10^{14} \mathrm{~W} . \mathrm{cm}^{-2}$ in the middle of a supersonic Helium jet with a $1 \mathrm{~mm}$ diameter opening. Due to gas expansion, we observe, using the interferometry diagnostic (see below), that the effective interaction length in the plasma is $\sim 1.5 \mathrm{~mm}$. The pressure of the jet was varied to modify the background electron density. Before focusing, the $90 \mathrm{~mm}$ diameter interaction laser beam was split evenly in a Mach-Zehnder interferometer to produce two replicas of the beam with slight angular separation of at most $0.15 \mathrm{mrad}$. When focused, this resulted in two spots separated by a variable and controllable distance. The MachZehnder was set such that the two output beams were synchronized. The beams, which were focused using an $f / 24(\mathrm{f}=2.1 \mathrm{~m})$ lens, were wavefront-corrected before every shot using adaptative optics [16]. This resulted in a focal spot of $60 \mu \mathrm{m}$ FWHM for each beam (corresponding to twice the diffraction limit) with $\sim 30 \%$ of the beam energy within the central lobe of the focal spot. The Rayleigh length of $1.9 \mathrm{~mm}$ was larger than the interaction length in the jet $(\sim 1.5 \mathrm{~mm})$. The on-axis transmitted light was collected through an $f / 6$ large aperture lens. Visible light was rejected from the detector by optical filters. The focal point of the laser was imaged onto a high-speed 2D spatially resolved sampling camera (HISAC) [38] composed of a fiber optics bundle coupled to an optical streak camera. The bundle was tapered at its other end in a 1D line which was aligned onto the slit of the streak camera. At the output of the streak camera, the time-swept 1D image (i.e. each line of the swept signal) was reorganized for each time so that the $2 \mathrm{D}$ image was recomposed. This way, the HISAC diagnostic obtained a sequence of time-resolved 2D images with a temporal resolution limited by the streak camera (30 ps) and a spatial resolution limited by the streak camera, the fiber size and the optical image-relay system. The field of view and the effective resolution, in the gas jet equivalent plane, were chosen to be $550 \times 600 \mu \mathrm{m}$ and $\sim 60 \mu \mathrm{m}$ respectively. A frequency-doubled (at $\lambda=528$ $\mathrm{nm}$ ) low-energy short pulse (400 fs FWHM) probe beam crossed the gas jet perpendicular to the interaction beams. The image of the interaction region was then sent through a Nomarski interferometer. The resulting phase maps of the interaction allowed observation of the density channels created by the long pulse beams [21].

The 2D simulations of the plasma evolution are performed using the multidimensional hydrodynamics CHIC code [35-37] which allows numerical simulations of high-power laser interaction with matter. This 
2D planar/axisymetrical code is based on both original physical models and numerical schemes. The hydrodynamic scheme is based on a second order cell-centered Lagrangian scheme [39]. The extension to the arbitrary Lagrangian Eulerian (ALE) has been implemented in order to improve accuracy, robustness, and computational efficiency of the calculation. Modelling of heat conduction uses an original high order cell-centered diffusion scheme on an unstructured mesh [40]. This code includes detailed physics for numerous phenomena including hydrodynamics, electron and ion conduction, thermal coupling and detailed radiation transport. A non-local electron transport model has been developed as well in CHIC [41]. It was used for the simulations performed in the present study. We checked that an alternative fluxlimited Spitzer-Härm [36] scheme for the heat transport (with a flux limiter at 10\%) could reproduce the hydrodynamic evolution observed when using the non-local transport scheme. Note however that in general a flux-limited scheme is physically less valid as the flux-limiter value needs to be adapted to each peculiar physical situation. Ionisation, equation of state and opacity data are tabulated, assuming local thermodynamic equilibrium (LTE) or non-LTE model. Inverse Bremsstrahlung laser heating is taken into account.

Regarding the 2D autocorrelation calculations, we calculate the 2D spatial autocorrelation function of the time-resolved 2D images of the beam spatial transverse profile as $A(\rho)=\int I(\mathbf{r}) I(\mathbf{r}+\boldsymbol{\rho}) \mathrm{d} \mathbf{r}$ where $\mathrm{I}(\mathbf{r})$ is the time-resolved $2 \mathrm{D}$ image and $\mathrm{A}(\boldsymbol{\rho})$ is the resulting $2 \mathrm{D}$ autocorrelation. This is done using Fourier transforms (Wiener-Khinchin theorem). It is then used to measure the azimuthallyaveraged FWHM of the autocorrelation function. For well-separated beams, this calculation yields the individual beam size; for merged beams it yields the size of the overall beam. This is compared to the size that would have resulted from the simple addition of individual beams. For this, we used the images resulting from single beam propagation and added the image to itself with a determined separation. As the single beam size varies with time (see Fig. 1(b)), this was done for each temporal frame. We then calculated the size of this composite image using the same autocorrelation procedure. 
FIGURES CAPTIONS
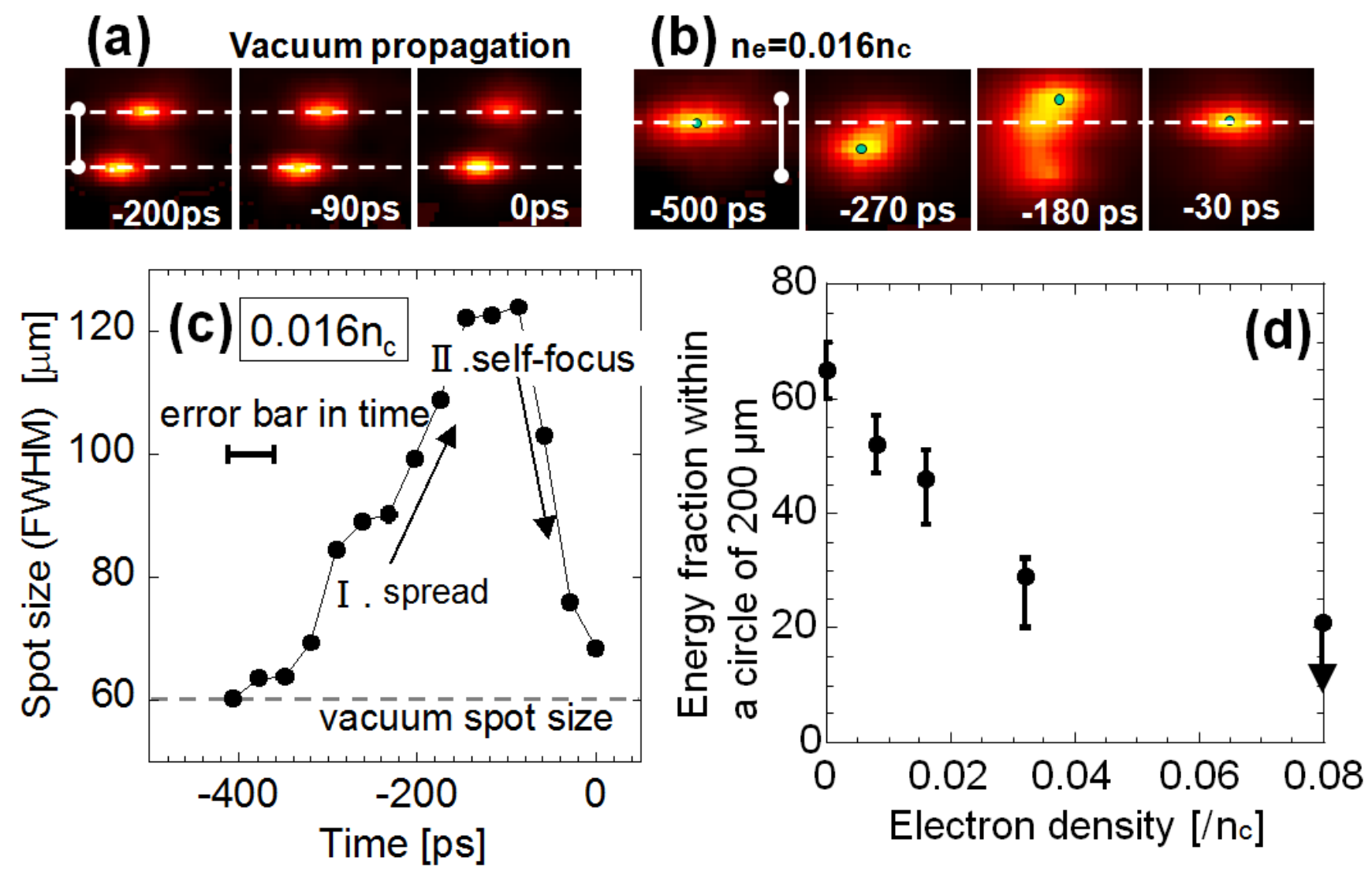

Figure 1: Delocalization of a high-power single laser beam propagating through a low-density plasma compared to stable propagation in vacuum. (a) Time-resolved 2D beam spatial distributions of two high-power parallel beams focused in vacuum. 0 ps indicates the temporal peak of the pulse. The vertical scale bar in the image at -200 ps indicates $150 \mu \mathrm{m}$. Each 2D transverse beam image is integrated over $30 \mathrm{ps}$ and is rescaled in brightness to the maximum intensity within the image. The beam location, as expected, does not change in the transverse plane, as can be seen from the fixed position of the beam centroid with respect to the white dashed line. (b) Same, but for a single beam propagating through a $0.016 n_{c}$ plasma. The vertical bar indicates $150 \mu \mathrm{m}$. The green dot locates the point of peak intensity in each image. It oscillates with respect to the incident beam location (still represented by the white dashed line). We concentrate here on vertical oscillations since when we put two beams (see (a) or Fig.2), they will be arranged side-by-side vertically. (c) Temporal evolution of the beam spot size for a single beam propagating in same $0.016 \mathrm{n}_{\mathrm{c}}$ plasma. (d) Beam energy fraction within a 
$200 \mu \mathrm{m}$ diameter circle vs. electron density at the temporal peak of the pulse $(\mathrm{t}=0)$. The error bars come from the standard deviation of values from shot to shot. 


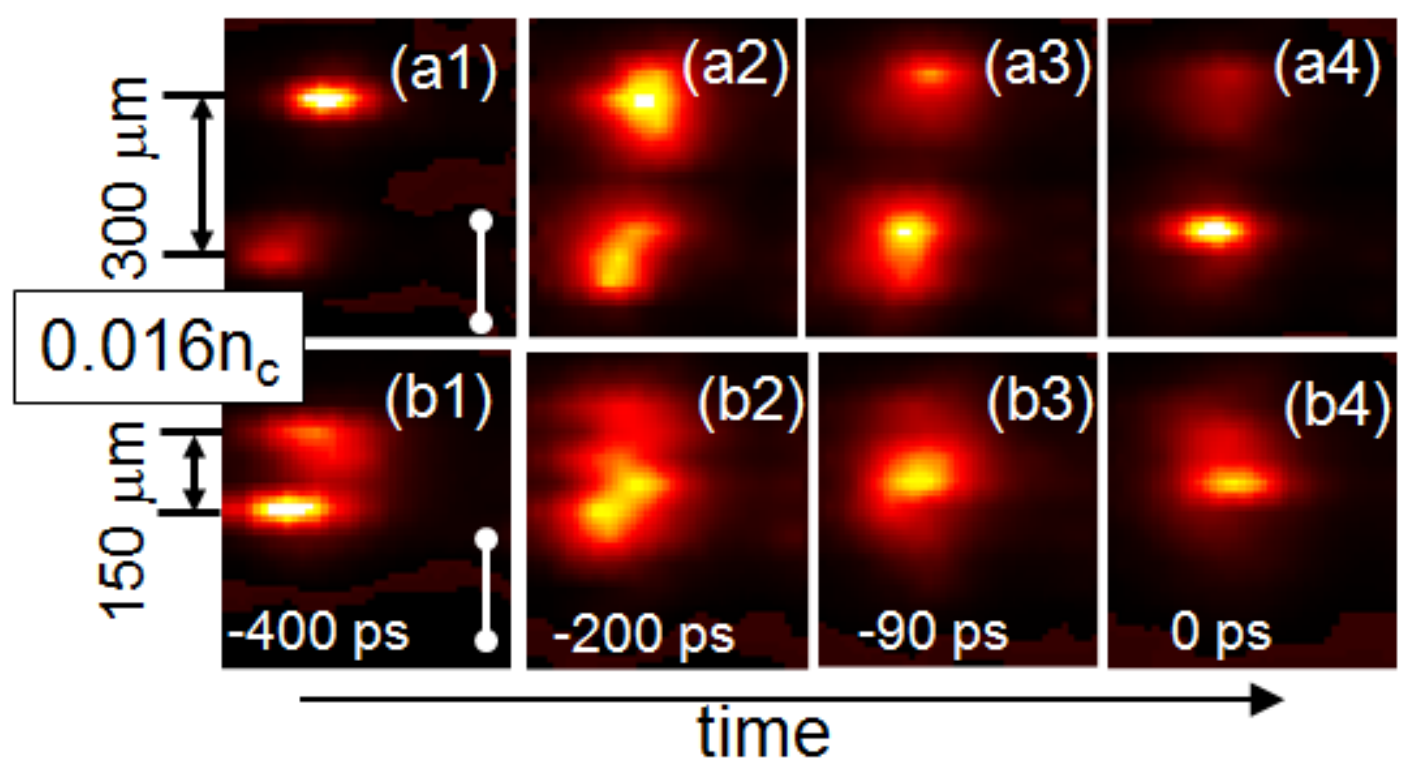

Figure 2: Temporal dynamics of two parallel beams propagating in a $0.016 n_{c}$ plasma for two initial separation distances. Each 2D image is integrated over 30 ps and rescaled in brightness to the maximum intensity within the image. The vertical scale bar in the image at -400 ps indicates $200 \mu \mathrm{m}$. 



Figure 3: Plasma channels expansion allowing or not the formation of a common waveguide for two laser beams to merge. (a-b) Interferograms measured -127 ps before the peak of the pulse reaches the gas jet. The background ionized electron density is $0.016 \mathrm{n}_{\mathrm{c}}$ in both cases. (a) and (b) have been acquired simultaneously as Fig.2(a) and Fig.2(b) (using (a) $300 \mu \mathrm{m}$ and (b) $150 \mu \mathrm{m}$ separation between the beams). The dashed lines are guides for the eyes following the interference fringes. (c) Density map at the middle of the channel obtained from (b) by Abel inversion. Abel inversion cannot be performed for (a) since it is not axially symmetric. (d) Ray-tracing calculation performed using the refractive index profile given by the density depletion shown in (c). The gray scale encodes the refractive index (darker zones correspond to higher refractive index). Ray propagation is from left to right. The rays are initially 
horizontal (corresponding to a plane wave at the focus). The ray indicating the initial location of the FWHM of the envelope formed by the two beams corresponds to the value calculated in Fig.6(a). 

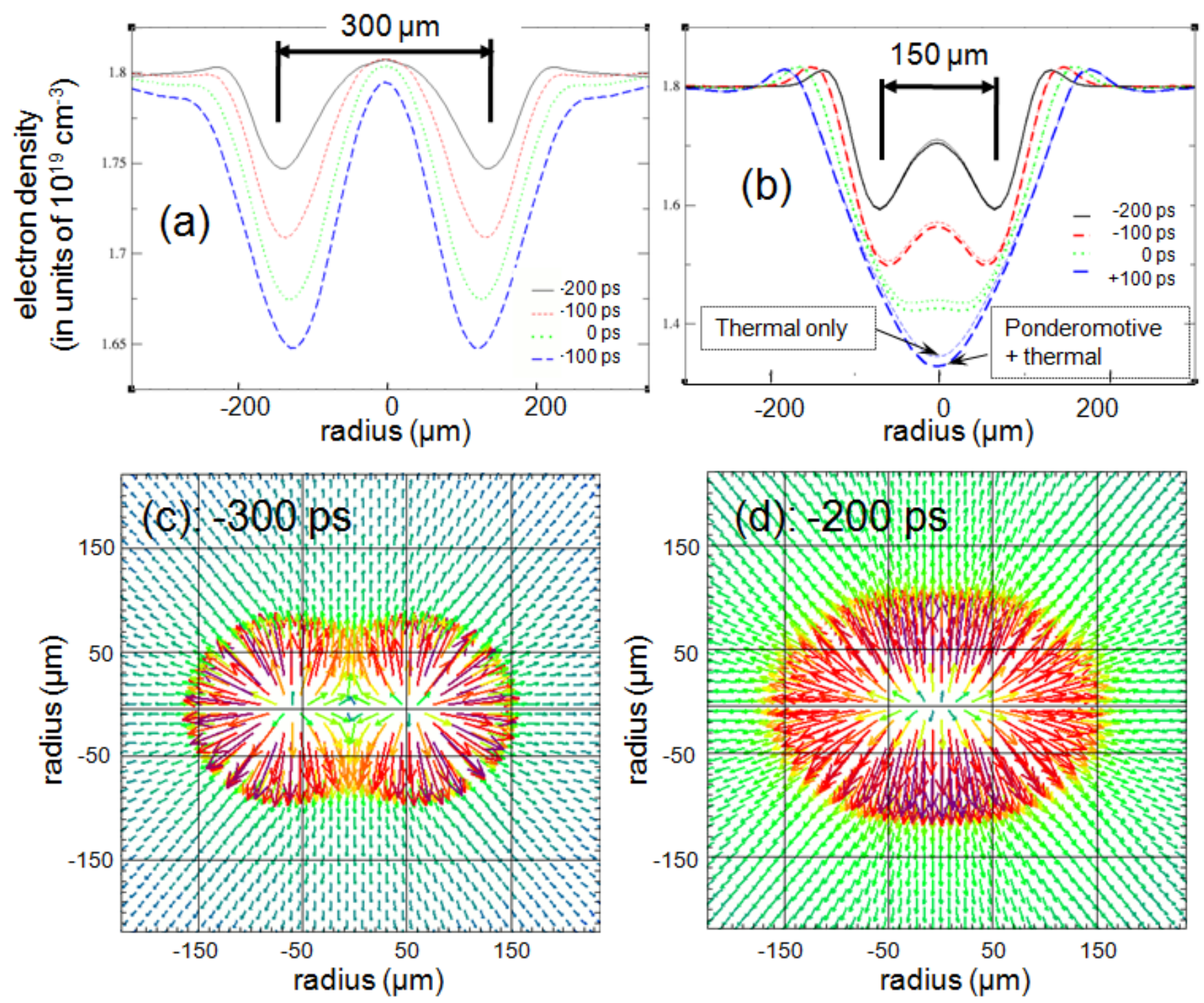

Figure 4: 2D CHIC simulations of the plasma hydrodynamic evolution in a plane transverse to the beam propagation showing the mechanism leading to plasma channel merging. (a-b) Lineouts of the electron density along the beam separation and at different times. The two simulations are performed using (a) $300 \mu \mathrm{m}$ and (b) $150 \mu \mathrm{m}$ separation between the beams. The laser beam parameters are otherwise the same as in Fig.2 and the initial electron plasma density is $0.018 \mathrm{n}_{\mathrm{c}}$. The spreading (according to Fig.1.c) and motion (according to Fig.1.b) of each beam is taken into account in the simulation. Time 0 refers to the temporal peak of the pulses. (c-d) 2D maps of the plasma speed (encoded by the length, color code and direction of the arrows) given by 2D CHIC simulations of the plasma hydrodynamic evolution in the plane transverse to the beam propagation. The two maps correspond to two different times, as indicated, and otherwise to the same conditions as in (b), i.e. to a $150 \mu \mathrm{m}$ separation between the laser beams. On (c) one can see that the plasma motion between the two laser axes is deflected and not accumulated at the 
mid-point between the two beams, with the plasma being expelled toward the edges of the focal spots. 




$-400 \mathrm{ps}$

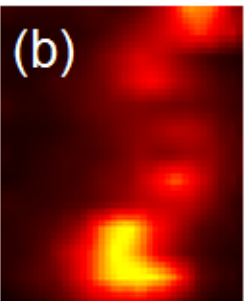

-200 ps

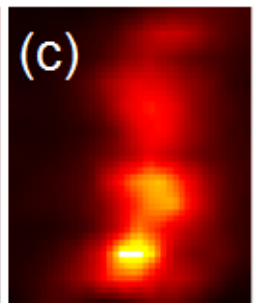

$-90 \mathrm{ps}$

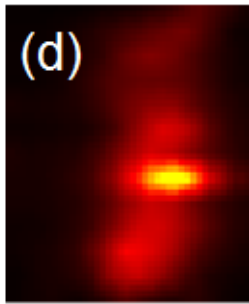

0 ps

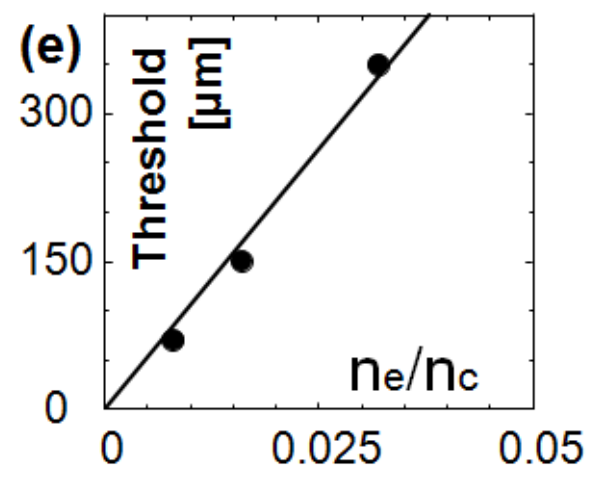

Figure 5: Behaviour of two beams coupling when increasing the plasma density. (a-d) Temporal dynamics of two parallel beams in a $0.032 n_{c}$ plasma for a $d=350 \mu \mathrm{m}$ initial separation. The scale bar indicates $200 \mu \mathrm{m}$. Each 2D image is integrated over $30 \mathrm{ps}$ and rescaled in brightness to the maximum intensity within the image. (e) Observed maximum distance (threshold) between two beams for beam merging to take place as a function of plasma density. 


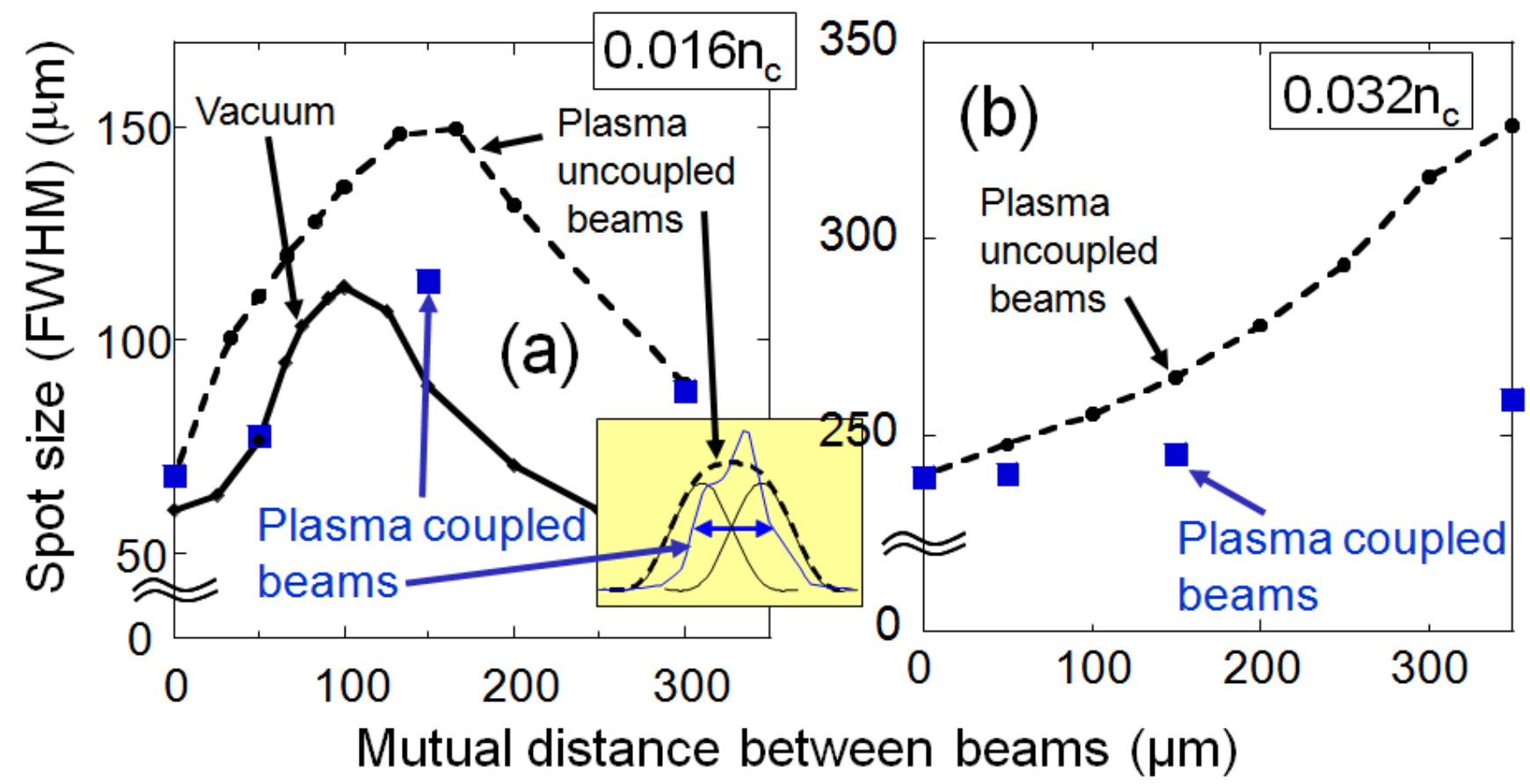

Figure 6: Quantitative measurement of beam coupling in plasma as a function of the mutual distance between the two beams and the plasma density. (a) Autocorrelated spot size measured at the peak of the pulse from time-resolved images. The boxes labeled "plasma coupled beams" correspond to experimentally observed images using two parallel beams propagated in plasma with various beam separation values. The background ionized electron density is for (a) $0.016 n_{c}$. For this we used, for example, images as shown in Fig.2 and 3. The dashed line with dots labeled "plasma uncoupled beams" corresponds to the case of synthetic images obtained by mathematically adding the image of a single plasma-propagating beam (i.e. propagating in the same conditions, in the same plasma, see Fig.1(b)) to itself, using a variable spatial shift between the two. The black line with diamonds labeled "vacuum" corresponds to the case of two linearly-diffracting (i.e. propagated in vacuum) beams with a variable separation. (b) Same but for a background ionized electron density of $0.032 n_{c}$. Here the "vacuum" case, which is identical to the one shown in (a), is not presented as the spot size values are much lower than the ones recorded for the plasma experiment. 


\section{REFERENCES}

1 Stegeman, G. and Segev, M. Optical Spatial Solitons and Their Interactions: Universality and Diversity. Science 286, 1518-1523 (1999).

2 Meng, H., Salamo, G., Shih, M. and Segev, M. Coherent collisions of photorefractive solitons. Opt. Lett. 22, 448-450 (1997).

3 Krolikowski, W. and Holmstrom, S. Fusion and birth of spatial solitons upon collision. Opt. Lett. 22, 369-371 (1997).

4 Shih, M. and Segev, M. Incoherent collisions between two-dimensional bright steady-state photorefractive spatial screening solitons. Opt. Lett. 21, 1538-1540 (1996).

5 Synder, A. W. and Mitchell, D. J. Accessible Solitons. Science 276, 1538-1541 (1997).

6 Rotschild, C., Alfassi, B., Cohen, C. and Segev, M. Long-range interactions between optical solitons. Nat. Phys. 2, 769-774 (2006).

7 Tzortzakis, S. et al. Breakup and Fusion of Self-Guided Femtosecond Light Pulses in Air. Phys. Rev. Lett. 86, 5470 - 5473 (2001).

$8 \mathrm{Xi}$, T. et al. Interaction of Light Filaments Generated by Femtosecond Laser Pulses in Air. Phys. Rev. Lett. 96, 025003 (2006).

9 Kruer, W. Ponderomotive and thermal filamentation of laser light. Comments Plasma. Phys. Controlled Fusion 9, 63-72 (1985).

10 Tikhonchuk, V. T. et al. Stimulated Brillouin and Raman scattering from a randomized laser beam in large inhomogeneous collisional plasmas. II. Model description and comparison with experiments. Phys. Plasmas 8, 1636-1649 (2001).

11 Rose, H. A. and DuBois, D. F. Laser hot spots and the breakdown of linear instability theory with application to stimulated Brillouin scattering. Phys. Rev. Lett. 72, 2883-2886 (1994).

12 Hüller, S. et al. Interaction of two neighboring laser beams taking into account the effects of plasma hydrodynamics. Phys. Plasmas 4, 2670-2680 (1997).

13 Ren, C. et al. On the mutual interaction between laser beams in plasmas. Phys. Plasmas 9, 23542363 (2002).

14 Shukla, P. et al. Instability and dynamics of two nonlinearly coupled laser beams in a plasma. Phys. Plasmas 13, 053104 (2006). 
15 Williams, E. On the control of filamentation of intense laser beams propagating in underdense plasma. Phys. Plasmas 13, 056310 (2006).

16 Wattellier, B. et al. Generation of a single hot spot by use of a deformable mirror and study of its propagation in an underdense plasma. J. Opt. Soc. Am. B. 20, 1632 (2003).

17 Young, P. et al. Observations of Laser-Beam Bending due to Transverse Plasma Flow. Phys. Rev. Lett. 81, 1425-1428 (1998);

18 Montgomery, D. et al. Flow-Induced Beam Steering in a Single Laser Hot Spot. Phys. Rev. Lett. 84, 678-681 (2000).

19 Labaune, C. et al. Laser-plasma interaction in the context of inertial fusion: experiments and modelling. Eur. Phys. J. D 44, 283-288 (2007).

20 Denavit, J.and Phillion, D. W. Laser ionization and heating of gas targets for long-scale-length instability experiments. Phys. Plasmas 1, 1971-1984 (1994).

21 de Wispelaere, E. et al. Formation of plasma channels in the interaction of a nanosecond laser pulse at moderate intensities with helium gas jets. Phys. Rev. E 59, 7110-7120 (1999).

22 Malka, V. et al. Strong self-focusing in quasi-stationary laser plasmas. Phys. Plasmas 7, 42594265 (2000).

23 E. J. Valeo and Estabrook, K. G. Stability of the Critical Surface in Irradiated Plasma, Phys. Rev. Lett. 34, 1008 (1975).

24 Pesme, D. etal. Resonant Instability of Laser Filaments in a Plasma. Phys. Rev. Lett. 84, 278-281 (2000).

25 Michel, P. et al. Studies of the laser filament instability in a semicollisional plasma. Phys. Plas$\operatorname{mas}$ 7, 3545-3553 (2003).

26 Myatt, J. et al. Nonlinear Propagation of a Randomized Laser Beam through an Expanding Plasma. Phys. Rev. Lett. 87, 255003 (2001).

27 Johnson, L. and Chu, T. Measurements of electron density evolution and beam self-focusing in a laser-produced plasma. Phys. Rev. Lett. 32, 517-520 (1974).

28 Fuchs, J. et al. Experimental Evidence of Plasma-Induced Incoherence of an Intense Laser Beam Propagating in an Underdense Plasma. Phys. Rev. Lett. 86, $432-435$ (2001). 
29 Epperlein, E.M., Kinetic theory of laser filamentation in plasmas. Phys. Rev. Lett. 65, 2145 2148 (1990).

30 Epperlein, E. M. and Short, R. W. Nonlocal heat transport effects on the filamentation of light in plasmas. Phys. Fluids B 4, 2211-2216 (1992).

31 Berger, R. L., Valeo, E. J., Brunner, S. The transition from thermally driven to ponderomotively driven stimulated Brillouin scattering and filamentation of light in plasma. Phys. Plasmas 12, 062508 (2005).

32 Chessa, P. et al. Temporal and Angular Resolution of the Ionization-Induced Refraction of a Short Laser Pulse in Helium Gas. Phys. Rev. Lett. 82, 552-555 (1999).

33 Mitchell, M., Chen, Z., Shih, M. and Segev, M. Self-trapping of partially spatially incoherent light. Phys.Rev. Lett. 77, 490-493 (1996).

34 Kruer, W. L. Nonlinear estimates of Brillouin scatter in plasma. Phys. Fluids 23, 1273-1275 (1980).

35 Pesme, D. et al. Laser-plasma interaction studies in the context of megajoule lasers for inertial fusion. Plasma Phys. Control. Fusion 44, B53-B67 (2002).

36 Atzeni, S., and Meyer-ter-Vehn, J. The Physics of Inertial Fusion. Oxford University Press (2009).

37 Glenzer, S. et al. Experiments and multiscale simulations of laser propagation through ignitionscale plasmas. Nature Phys. 3, $716-719$ (2007).

38 Kodama, R. et al. Development of a two-dimensional space-resolved high speed sampling camera. Rev. Sci. Inst. 70, 625-628 (1999).

39 Maire, P.-H., Abgrall, R., Breil, J. and Ovadia, J. A Cell-Centered Lagrangian Scheme for TwoDimensional Compressible Flow Problems. SIAM Journal of Scientific Computing 29, 1781-1824 (2007).

40 Breil, J., and Maire, P.-H. A cell-centered diffusion scheme on two-dimensional unstructured meshes. J. of Comp. Phys. 224, 785-823 (2007).

41 Schurtz, G. et al. Revisiting Nonlocal Electron-Energy Transport in Inertial-Fusion Conditions. Phys. Rev. Lett. 98, 095002 (2007). 